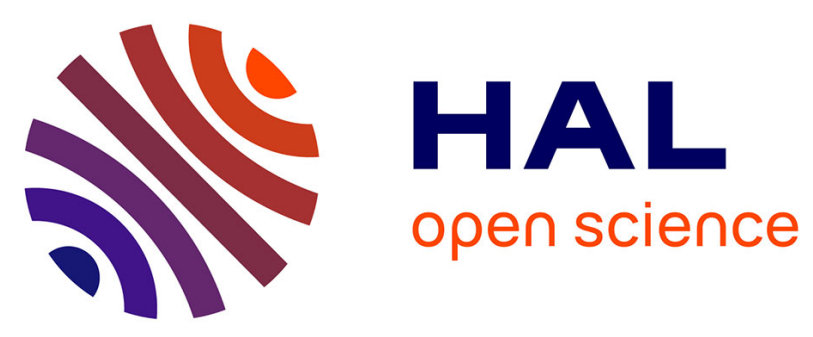

\title{
Somatosensory temporal discrimination in patients with primary focal dystonia
}

Alessandra Scontrini, Antonella Conte, Giovanni Defazio, Mirta Fiorio, Giovanni Fabbrini, Antonio Suppa, Michele Tinazzi, Alfredo Berardelli

\section{To cite this version:}

Alessandra Scontrini, Antonella Conte, Giovanni Defazio, Mirta Fiorio, Giovanni Fabbrini, et al.. Somatosensory temporal discrimination in patients with primary focal dystonia. Journal of Neurology, Neurosurgery and Psychiatry, 2009, 80 (12), pp.1315. 10.1136/jnnp.2009.178236 . hal-00552785

\section{HAL Id: hal-00552785 \\ https://hal.science/hal-00552785}

Submitted on 6 Jan 2011

HAL is a multi-disciplinary open access archive for the deposit and dissemination of scientific research documents, whether they are published or not. The documents may come from teaching and research institutions in France or abroad, or from public or private research centers.
L'archive ouverte pluridisciplinaire HAL, est destinée au dépôt et à la diffusion de documents scientifiques de niveau recherche, publiés ou non, émanant des établissements d'enseignement et de recherche français ou étrangers, des laboratoires publics ou privés. 


\section{Somatosensory temporal discrimination in patients with primary focal dystonia}

Alessandra Scontrini ${ }^{1}(\mathrm{MD})$, Antonella Conte ${ }^{1}(\mathrm{MD})$, Giovanni Defazio ${ }^{2}$ (MD), Mirta

Fiorio $^{3}(\mathrm{MD})$, Giovanni Fabbrini ${ }^{1}(\mathrm{MD})$, Antonio Suppa ${ }^{1}(\mathrm{MD})$, Michele Tinazzi ${ }^{3,4}$ (MD),

Alfredo Berardelli ${ }^{1}(\mathrm{MD})$

${ }^{1}$ Department of Neurological Sciences and Neuromed Institute, "Sapienza”, University of Rome

${ }^{2}$ Institute of Neurology, University of Bari, Italy

${ }^{3}$ Department of Neurological and Vision Sciences, Section of Rehabilitative Neurology, University of Verona

${ }^{4}$ Neurology Unit, Borgo Trento Hospital, Verona

Key words: dystonia, somatosensory temporal discrimination

Manuscript word count: 3471

The Corresponding Author has the right to grant on behalf of all authors and does grant on behalf of all authors, an exclusive licence on a worldwide basis to the BMJ Publishing Group

Ltd and its Licensees to permit this article (if accepted) to be published in JNNP.

\section{Corresponding author:}

Prof. Alfredo Berardelli

Department of Neurological Sciences

"Sapienza", University of Rome

Viale dell'Università, 30, 00185 Rome, Italy

Telephone number: 0039-06-49914700

Fax: 0039-06-49914302

E-mail: alfredo.berardelli@uniroma1.it 


\section{ABSTRACT}

Purposes: To determine whether somatosensory temporal discrimination will reliably detect subclinical sensory impairment in patients with various forms of primary focal dystonia. Methods: We tested the somatosensory temporal discrimination threshold (STDT) in 82 outpatients affected by cranial, cervical, laryngeal and hand dystonia. Results were compared with those for 61 healthy subjects and 26 patients with hemifacial spasm, a non-dystonic disorder. The STDT was tested by delivering paired stimuli starting with an interstimulus interval of 0 msec followed by a progressively increasing interstimulus interval.

Results: STDT was abnormal in all the different forms of primary focal dystonias in all three body regions (eye, hand and neck), regardless of the distribution and severity of motor symptoms. Receiver operating characteristic curve analysis calculated in the three body regions yielded high diagnostic sensitivity and specificity for STDT abnormalities.

Conclusions: these results provide definitive evidence that STDT abnormalities are a generalized feature of patients with primary focal dystonias and are a valid tool for screening subclinical sensory abnormalities. 


\section{INTRODUCTION}

Although dystonia is mainly characterized by motor disorders some patients also manifest sensory symptoms.[1] For instance, patients with blepharospasm often complain of dry eyes and patients with hand dystonia may have an impairment of graphesthesia, kinesthesia and stereognosis.[2-4]

Sensory abnormalities have been demonstrated in patients with focal dystonia and in asymptomatic relatives using the grating orientation tasks [5-8], which assess somatosensory spatial discrimination. Another way to investigate sensory system is to test the somatosensory temporal discrimination (STD). STD is the ability to perceive as temporally separate two successive tactile stimuli applied to a body part. STD plays a basic role in sensory integration and depends on the integrity of specific sensory systems together with that of other structures involved in the temporal processing of tactile stimuli, including the basal ganglia.[9] STD can be measured by computing its threshold (STDT), defined as the shortest interval elapsing between two stimuli presented separately in time and perceived as separated. Earlier studies documented increased STDT values in the hands of small groups of patients with generalized, hand, cervical and cranial dystonia.[7,10-15] These findings raised the possibility that the STDT could be abnormal both in affected and unaffected body parts. To date, however, no study has systematically explored STDT in several distant dystonic and non-dystonic body parts in a large sample of patients with various focal dystonias. Nor have previous studies assessed the reliability of STDT testing. This information is crucial to detect subclinical sensory impairment in patients with dystonia, and in understanding how abnormal STDT is related to the substrate on which dystonia develops, regardless of the variable phenotype. In this study we investigated STDT in a population of 82 patients with various forms of primary focal dystonia -- cranial (BS), cervical (CD), hand (HD) and laryngeal dystonia (LD) -- and in each group we systematically investigated STD in three distant dystonic and nondystonic body parts (eye, neck and hand). We compared the results with those from healthy 
subjects and patients with hemifacial spasm (HFS) -- a non-dystonic disorder characterised by involuntary muscle contractions of peripheral origin.

\section{METHODS}

\section{Subjects}

We recruited from the outpatient clinic of the Department of Neurological Sciences, Sapienza, University of Rome, 82 consecutive patients with various forms of primary focal dystonia (35 with BS; 30 with CD; 8 with HD; and 9 with LD) (Table 1). For comparison we recruited 61 healthy subjects and 26 patients with HFS. Patients with secondary dystonias were excluded by means of medical history, neurological examination and neuroimaging investigations. All patients had received treatment with botulinum toxin until 4-5 months before the study and when studied had dystonic symptoms. All subjects gave their written informed consent before participating in the study. The procedure was approved by the ethical committee of the Department of Neurological Sciences, Sapienza, University of Rome and conformed with the Declaration of Helsinki. 
TABLE 1: Patients with focal dystonias

\begin{tabular}{|l|l|l|l|l|l|}
\hline Diagnosis & No. patients & Women, No. & Age, y & Disease duration, y & Severity, y \\
& & & mean \pm SE & mean \pm SE & mean \pm SE \\
\hline BS & 35 & 24 & $67.7 \pm 1.74$ & $10.6 \pm 1.2$ & \\
\hline CD & 30 & 22 & $40-80$ & & $2 \pm 0.1$ \\
\hline HD & 8 & 1 & $55.6 \pm 2.4$ & $13.6 \pm 2.0$ & $13.3 \pm 0.8$ \\
& & & $32-80$ & & $5.0 \pm 0.5$ \\
\hline LD & 9 & 5 & $56.7 \pm 5.3$ & $15.1 \pm 4.6$ & \\
\hline
\end{tabular}

\section{Patients with blepharospasm}

The study group comprised 35 patients ( 24 women and 11 men; mean age: $67.7 \pm 1.74$ years, range 40-80 years, mean education level: $6.9 \pm 0.8$ years) with focal BS. Disease lasted on average $10.6 \pm 1.2$ years (Table 1 ). A single examiner assessed the severity of motor impairment on a three-point clinical scale $(1=$ mild to $3=$ severe impairment $)$ [15]. These patients' performance was compared with that of 35 age-matched control subjects (16 women and 19 men, mean age $65 \pm 1.7$ years, range 42-83). Educational level did not differ between patients and age-matched healthy subjects $(\mathrm{p}=0.14)$.

Patients with cervical dystonia

This group comprised 30 patients with focal CD ( 22 women and 8 men; mean age: $55.6 \pm 2.4$ years, range $32-80$ years, mean education level: $10.5 \pm 0.9$ years). Disease lasted on average $13.6 \pm 2.0$ years. The severity of motor impairment was assessed with the Toronto Western 
Scontrini et al. 6

Spasmodic Torticollis Rating Scale (TWSTRS)(Table 1).[16] These patients' performance was compared with that of 30 age-matched control subjects (16 women and 14 men, mean age $55 \pm 2.3$ years, range $32-81$ ). Educational level did not differ between patients and age-matched healthy subjects $(\mathrm{p}=0.8)$.

Patients with hand dystonia

This group comprised 8 patients (one woman and seven men; mean age: $56.7 \pm 5.3$ years, range 33-82 years, mean education level: $12.3 \pm 0.6$ years). All patients had dystonia involving the right side (dominant hand). Five had writer's cramp only, one also had cranial dystonia and the other two also had CD. HD lasted on average $15.1 \pm 4.6$ years. The severity of motor impairment was assessed with the Burke-Fahn Marsden Movement and Disability Scale (Table 1).[17] These patients' performance was compared with that of 8 age-matched control subjects ( 4 women and 4 men, mean age $56 \pm 5.1$ years, range 34-81). Educational level did not differ between patients and age-matched healthy subjects $(\mathrm{p}=0.32)$.

\section{Patients with laryngeal dystonia}

The LD group comprised 9 patients ( 5 women; mean age: $50.4 \pm 5.7$ years, range $34-76$ years, mean education level: $12.6 \pm 6.2$ years. Five patients had LD as the only symptom, two also had CD and two had writer's cramp. LD lasted on average $12.8 \pm 7.6$ years. The severity of vocal impairment was assessed with a three-point clinical scale $(1=$ mild to $3=$ severe impairment) (Table 1). These patients' performance was compared with that of 9 age-matched

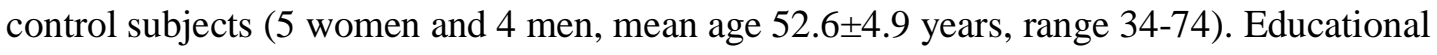
level did not differ between patients and age-matched healthy subjects $(\mathrm{p}=1)$.

\section{Patients with hemifacial spasm}

As control group for BS patients, we also tested 26 patients with primary HFS (15 women and 11 men; mean age: $62.2 \pm 2.6$ years, range 40-80 years, mean education level: $9.0 \pm 0.8$ years): 10 had HFS on the right side and 16 on the left side. Disease duration was on average 
$9.4 \pm 1.0$ years. The severity of motor impairment was assessed on a three-point clinical scale $(1.5 \pm 0.1 ; 1=$ mild to $3=$ severe impairment $) .[15]$

\section{Stimuli and STD procedure}

Somatosensory discrimination was tested by delivering paired stimuli starting with an interstimulus interval (ISI) of $0 \mathrm{msec}$ (simultaneous pair), followed by progressively increasing ISI (in $10 \mathrm{msec}$ steps). Paired tactile stimuli consisted of square-wave electrical pulses delivered with a constant current stimulator (STM 140; HTL, Udine, Italy) through surface skin electrodes with the anode located $0.5 \mathrm{~cm}$ distally from the cathode. The stimulation intensity was determined for each subject by delivering series of stimuli at an increasing intensity from $2 \mathrm{~mA}$ in steps of $1 \mathrm{~mA}$. The minimal intensity perceived by the subject in 10 of 10 consecutive stimuli was used in the experimental test. The surface skin electrode was applied to three different body regions: the index finger (hand), near the orbit (eye) and to the neck, in a single experimental session and in randomized order across subjects.[7,11-15] Because dystonia differentially affected these body districts in 75 of the 82 patients STDT was tested in three body regions (one body part affected by motor symptoms and two body regions unaffected). In 7 of the 82 patients (one HD patient also had cranial dystonia, two HD patients also had CD, two LD patients also had HD and two LD patients also had CD) STDT was tested in two affected body regions and in one unaffected body region. The affected body region was tested bilaterally (i.e. ipsilaterally and controlaterally to the location of the motor symptom, in randomized order across subjects). Subjects had to report whether they perceived a single stimulus or two temporally separated stimuli. The first of three consecutive ISI at which participants recognized the stimuli as temporally separated was considered the STDT. To check that subjects maintained attention levels constant during the test and to minimize the risk of a response bias, the STD testing procedure included trials consisting of a single stimulus every ten pairs. 
For healthy subjects and patients with HFS, STDT was assessed in three areas on the same body side (eye, neck, and hand). The order of presentation and side of stimulation were randomly assigned. Each session comprised four separate blocks. The STDT for each stimulated body site was defined as the average of four STDT values, one for each block, and was entered in the data analysis.

\section{Control experiments}

To rule out the possible influence of muscular activity on the STDT, in a subgroup of 12 healthy subjects (mean age: $51 \pm 3$ years) STDT was tested twice, at rest and during a voluntary muscle contraction. Throughout testing participants were therefore asked to maintain the head turned to one side, so as to reproduce a common CD posture.

\section{Statistical analysis}

Data obtained from each patient group were compared with those from a subgroup of healthy subjects selected from the total control group according to an age-matched criterion. The results of patients with blepharospasm were also compared with those obtained in patients with HFS.

Values are expressed as means and standard error (SE). STDT values in patients and healthy subjects were tested by means of separate repeated measures analyses of variance (ANOVA). Each ANOVA had the "Group" as between-subjects factor (BS vs. HFS vs. control subjects; CD vs. control subjects; HD vs. control subject; LD vs. control subjects), and "Body part" (eye, hand, and neck) as within-subjects factor. In HFS patients the STDT values from affected and unaffected sides were analyzed with a one-way ANOVA with "Side of stimulation" (affected vs. unaffected) as main factor. STDT values in the control experiments performed in a subgroup of healthy subjects were analyzed with a one-way repeated measures ANOVA with a within-subjects factor "session" (rest and contraction). The Spearman correlation coefficient was used for assessing possible relationships between the spasm 
Scontrini et al. 9

severity score, disease duration and STDT in patients groups. Tukey Honest Significance

Difference test was used for post hoc analysis. To see whether the degree of STDT alterations differs in the various forms of focal dystonia a between-group repeated measures ANOVA with "Body part" as main factor was performed.

To assess the diagnostic accuracy (sensitivity and specificity) of STDT testing in dystonia, we used a receiver operating characteristic (ROC) curve analysis. For STDT calculated in the different body regions (eye, hand, neck) we calculated the "cut-off value" corresponding to the highest diagnostic accuracy (minimal false-negative and false-positive results). The "cutoff value" was the point on the ROC curve, calculated by a dedicated software, that maximizes correct classifications of pathological conditions and minimizes errors. We also assessed the diagnostic sensitivity (proportion of patients with dystonia in whom STDT testing correctly yielded abnormal findings) (true positives) and specificity (proportion of control subjects in whom STDT testing correctly yielded normal findings (true negatives).

[18] To estimate the diagnostic accuracy of STD testing, we then calculated the area under the curve (AUC) value and the $z$ statistics.

\section{RESULTS}

All subjects received stimulation at comparable mean intensities $(\mathrm{p}=0.4)$.

\section{Patients with blepharospasm}

Repeated-measures ANOVA to compare differences in STDT values between patients with BS, HFS and healthy subjects showed a significant effect of the factor "Group" $\left(\mathrm{F}_{(2,93)}=16.1\right.$; $\mathrm{p}<0.001)$, a significant effect of the factor "Body part" $\left(\mathrm{F}_{(2,186)}=10.4 ; \mathrm{p}<0.001\right)$ but no significant interaction of the factors "Group" and "Body part" $\left(\mathrm{F}_{(4,186)}=0.49 ; \mathrm{p}=0.73\right)$. The significant effect of the factor "Body part" was due to higher thresholds for stimuli applied to the hand than to the eye and neck. Post hoc analysis showed that patients with BS had higher STDT values than patients with HFS and healthy subjects ( $\mathrm{p}<0.001$ for both comparisons). 
No significant difference was found between HFS and control subjects $(\mathrm{p}=0.9)$ and, in the HFS patients, between the affected and unaffected eye ( $\mathrm{p}=0.1)$ (Fig. 1)

Patients with cervical dystonia

The significant effect of the factor "Group" $\left(F_{(1,58)}=27.07 ; p<0.001\right)$, was due to the higher STDT in CD patients than in control subjects. The significant effect of the factor "Body part" $\left(F_{(2,116)}=9.90 ; p<0.001\right)$ was due to higher thresholds for stimuli applied to the hand than to the eye and neck. No significant interaction was found between the factors Group" and "Body part" $\left(\mathrm{F}_{(2,116)}=0.40 ; \mathrm{p}=0.66\right)($ Fig. 2$)$.

Patients with hand dystonia

The STDT was higher in patients with HD than in healthy subjects $\left(\mathrm{F}_{(1,14)}=5.64 ; \mathrm{p}=0.03\right)$. The significant effect of the factor "Body part" $\left(\mathrm{F}_{(2,28)}=4.60 ; \mathrm{p}=0.02\right)$ indicated higher STDT for stimuli applied to the hand than to the eye and neck. No significant interaction was found between the factors "Group" and "Body part" $\left(\mathrm{F}_{(2,28)}=0.54 ; \mathrm{p}=0.58\right)($ Fig. 3).

\section{Patients with laryngeal dystonia}

Repeated-measures ANOVA to compare the differences in STDT values between patients with LD and healthy subjects showed a significant effect of the factor "Group" $\left(\mathrm{F}_{(1,16)}=13.55\right.$; $\mathrm{p}=0.002$ ), with LD patients having higher STDT than control subjects, a significant effect of the factor "Body part" $\left(\mathrm{F}_{(2,32)}=4.88 ; \mathrm{p}=0.01\right)$, with higher STDT for stimuli applied to the hand than to the eye and neck, and no significant interaction between "Group" and "Body part" $\left(\mathrm{F}_{(2,32)}=0.10 ; \mathrm{p}=0.90\right)$ (Fig. 4).

Comparison between STDT alterations in the various forms of focal dystonia and clinical correlations

Between-group repeated measures ANOVA for STDT values comparing STDT alterations in the different forms of focal dystonia showed that factor "Group" was not significant $\left(\mathrm{F}_{(2,6)}=0.44 ; \mathrm{p}=0.84\right)$. 
No correlation was found between severity scores and STDT values and between disease duration and STDT values in any of the groups of patients tested.

\section{ROC curve analysis}

The ROC curve analysis showed that STDT calculated in the three body regions yielded high diagnostic specificity for the hand and the eye (hand: $82.8 \%$ and eye: $74.1 \%$ ) and high sensitivity for the three body regions tested (hand: $74.3 \%$; neck: $93.2 \%$ and eye: $90.5 \%$ ). The estimate of the AUC values (hand: 0.86; neck: 0.81; eye: 0.87 ) and the z statistics for the STDT in the three body regions (hand: 11.86; neck: 8.67; eye: 12.28) showed that STDT yielded accurate scores (fig.5).

\section{Control experiments}

In the subgroup of 12 healthy subjects in which STDT was tested twice, voluntary muscle contraction did not increase the STDT $\left(\mathrm{F}_{(2,22)}=0.70 ; \mathrm{p}=0.50\right)$.

\section{DISCUSSION}

Our study in patients with all the various forms of primary, focal dystonia shows abnormal STD in all three body regions tested, the eye, neck and hand. By systematically testing STD in the dystonic body part and in two non-dystonic body regions in patients with various forms of primary focal dystonia we confirm early reports performed in small group of patients with single forms of dystonia and we now provide definitive evidence that rather than being specific to the affected body region STDT abnormalities are a generalized feature in focal dystonias. The high diagnostic sensitivity and specificity STDT achieved in the ROC curve analysis suggests that STD is a reliable and valid tool for screening sensory abnormalities in patients with focal dystonias. 
Under our experimental conditions, STDT values differed significantly in dystonic patients and healthy subjects. Although we attribute these differences to the disease we cannot altogether exclude possible confounding effects, including for example attention. We nevertheless consider it unlikely that attention levels differed because we checked them continuously throughout testing. We can also practically exclude confounding from the involuntary muscle contraction present in patients with dystonia because our experiments testing healthy subjects during a voluntary contraction identified no differences in STDT values recorded at rest or during voluntary contraction. Two further points support this view: first, in patients with writer's cramp in whom involuntary contractions manifested only during writing, STDT testing detected abnormalities at rest; and second, the observation that in HFS, a non-dystonic condition characterized by involuntary muscle contractions of peripheral origin resembling those seen in patients with BS, the STDT is normal. An interesting finding that could extend current knowledge on the pathophysiology of sensory inputs in focal dystonia is that STDT testing disclosed abnormalities in all the patients we studied regardless of the distribution and severity of motor symptoms. Previous studies reported a correlation between the severity of writer's cramp and STDT alterations.[7,12,13] This finding was attributed to the more important role played by the sensory inputs in the pathophysiology of writer's cramp than in the other forms of primary dystonia. In line with our findings, however, Sanger et al., disclosed no correlation between the severity of writer's cramp and STDT alterations.[7] Our observation that the STDT abnormalities in HD patients who were tested while free of motor symptoms resembled those found in patients with the other forms of focal dystonia, further suggests that STDT abnormalities in focal dystonia are not directly caused by involuntary muscle contraction. Finally, the finding that STDT is abnormal in asymptomatic carriers of the DYT1 gene mutation, even those manifesting no dystonic activity, supports the hypothesis that STDT abnormalities are unrelated to the clinical manifestations of the disease.[19] 
Our findings are consistent with previous clinical, neurophysiological and neuroimaging studies demonstrating somatosensory abnormalities in primary dystonia. Several studies have demonstrated an abnormal somatotopy and disorganized receptive fields in sensory motor cortex in dystonia.[20,21] In healthy subjects, active movements reduce the amplitude of somatosensory evoked potentials (pre-movement gating) but in patients with hand dystonia pre-movement gating is absent suggesting an abnormality in the interaction between motor and sensory cortical areas.[22] Furthermore, studies investigating the inhibitory functions of the sensory system with the technique of somatosensory evoked potentials reported altered inhibitory functions within the somatosensory system, and in particular a loss of short latency inhibitory mechanisms in primary sensory cortex, leading to impaired afferent-input gating in dystonia.[23-24]. Afferent-input gating plays a role in the spatio-temporal separation of two incoming stimuli and allows information to be processed separately.[25] A deficit in this mechanism might lead to an STD deficit in primary dystonia.

Although our study leaves open the question of where the STD abnormalities related to dystonia arise, it again indicates the basal ganglia as the putative neural structures involved in the pathophysiology of the disease. The ability to discriminate stimuli as temporally separate is a complex process that involves several sensory system structures (parietal cortex) but also neural structures involved in timing functions including cerebellum and basal ganglia nuclei. As suggested by Mauk and Buonomano,[26] the cerebellum may act as a general-purpose interval timer in the range of tens to hundreds of ms. Animal experiments suggest that basal ganglia receive sensory information that plays a role in motor control and gates sensory inputs by selecting the information needed for guiding movement.[27] That basal ganglia abnormalities may lead to an alteration in the temporal processing of afferent signals is supported by the observation that STD abnormalities are also present in patients with Parkinson's disease [28]. The presence of STDT abnormalities in Parkinson's disease suggests that STDT abnormalities are not specific for dystonia. A functional MRI study 
reported basal ganglia and cerebellum activation during temporal and spatial tasks, whereas pre-SMA and anterior cingulated areas showed activity only during temporal testing.[29] The authors suggested that these cortical areas play a specific role within a basal ganglia-frontal cortex circuit in controlling temporal discrimination of somatosensory stimuli. Recent studies however, raised the hypothesis that also the cerebellum may play a role in the pathophysiology of dystonia.[30,31] A possible involvement of cerebellar circuits in the STDT alterations we found in patients with focal dystonia requires further studies.

By systematically testing STDT in different body regions we found that in healthy subjects and in patients with focal dystonia, regardless of the clinical form of focal dystonia STDT values were higher in the hand than in the face and neck. Our findings leave the physiological mechanisms underlying this body distribution pattern unknown.

In conclusion, with the experimental design we used in our large sample of patients, we found a widespread abnormality of STD in various types of primary focal dystonia in symptomatic and asymptomatic body parts independently from the clinical manifestations. These findings suggest that sensory abnormalities definitively play a role in the pathophysiology of dystonia and possibly also in spreading of dystonic symptoms in primary dystonia.[32] The possibility however, that sensory abnormalities may be a factor responsible for the spreading of dystonic symptoms needs to be addressed in further studies. In addition STD testing to seek sensory symptoms might be valuable in selecting asymptomatic relatives for genetic studies. 
Scontrini et al. 15

\section{Figure legends:}

Fig. 1 Somatosensory temporal discrimination threshold (STDT) in patients with blepharospasm (BS), hemifacial spasm (HFS) and healthy subjects. Differences in STDT values between BS patients and healthy subjects were statistically significant for the three body regions tested. 
Scontrini et al. 16

Fig. 2 Somatosensory temporal discrimination threshold (STDT) in patients with cervical dystonia (CD) and healthy subjects. Differences in STDT values between CD patients and healthy subjects were statistically significant for the three body regions tested. 
Scontrini et al. 17

Fig. 3 Somatosensory temporal discrimination thresholds (STDT) in patients with hand dystonia (HD). Differences in STDT values between HD patients and healthy subjects were statistically significant for the three body regions tested. 
Scontrini et al. 18

Fig. 4 Somatosensory temporal discrimination threshold (STDT) in patients with laryngeal dysphonia (LD) and healthy subjects. Differences in STDT values between LD patients and healthy subjects were statistically significant for the three body regions tested. 
Scontrini et al. 19

Fig. 5 The receiver operating characteristic (ROC) curve diagram for the three body regions tested in all dystonic patients. 


\section{References}

1. Berardelli A, Rothwell JC, Hallett $\mathrm{M}$ et al. The pathophysiology of primary dystonia. Brain 1998;121:1195-212.

2. Hallett M. Is dystonia a sensory disorder? Ann Neurol 1995;38:139-40.

3. Ghika J, Regli F, Growdon JH. Sensory symptoms in cranial dystonia: a potential role in the etiology? J Neurol Sci 1993;116:142-7.

4. Defazio G, Berardelli A, Hallett M. Do primary adult-onset focal dystonias share aetiological factors? Brain 2007;130:1183-93.

5. Molloy FM, Carr TD, Zeuner KE et al.. Abnormalities of spatial discrimination in focal and generalized dystonia. Brain. 2003;126:2175-82

6. Bara-Jimenez W, Shelton P, Hallett M. Spatial discrimination is abnormal in focal hand dystonia. Neurology. 2000;55:1869-73

7. Sanger TD, Tarsy D, Pascual-Leone A. Abnormalities of spatial and temporal sensory discrimination in writer's cramp. Mov Disord 2001;16:94-9.

8. O'Dwyer JP, O'Riordan S, Saunders-Pullman R, et al. Sensory abnormalities in unaffected relatives in familial adult-onset dystonia. Neurology 2005;65:938-40.

9. Tinazzi M, Rosso T, Fiaschi A. Role of the somatosensory system in primary dystonia. Mov Disord 2003;18:605-22.

10. Aglioti SM, Fiorio M, Forster B et al. Temporal discrimination of cross-modal and unimodal stimuli in generalized dystonia. Neurology 2003;60: 782-5.

11. Tinazzi M, Fiaschi A, Frasson E et al. Deficits of temporal discrimination in dystonia are independent from the spatial distance between the loci of tactile stimulation. Mov Disord 2002;17:333-8.

12. Bara-Jimenez W, Shelton P, Sanger TD et al. Sensory discrimination capabilities in patients with focal hand dystonia. Ann Neurol 2000;47:377-80. 
13. Fiorio M, Tinazzi M, Bertolasi L et al. Temporal processing of visuotactile and tactile stimuli in writer's cramp. Ann Neurol 2003;53:630-5.

14. Tinazzi M, Fiorio M, Bertolasi L et al. Timing of tactile and visuotactile events is impaired in patients with cervical dystonia. J Neurol 2004;25:85-90.

15. Fiorio M, Tinazzi M, Scontrini A, et al. Tactile temporal discrimination in patients with blepharospasm. J Neurol Neurosurg Psychiatry 2008;79:796-98.

16. Consky ES, Lang AE. Clinical assessments of patients with cervical dystonia. In: Jankovic J, Hallett M, editors. Therapy with Botulinum Toxin. New York, NY: Marcel Dekker, Inc.:1994;211-37.

17. Burke RE, Fahn S, Marsden CD et al. Validity and reliability of a rating scale for the primary torsion dystonias. Neurology 1985;35:73-7.

18. Pepe MS, Feng Z, Huang Y et al. Integrating the predictiveness of a marker with its performance as a classifier. Am J Epidemiol 2008;167:362-8.

19. Fiorio M, Gambarin M, Valente EM et al. Defective temporal processing of sensory stimuli in DYT1 mutation carriers: a new endophenotype of dystonia. Brain 2007;130:134-42.

20. Bara-Jimenez W, Catalan MJ, Hallett M et al. Abnormal somatosensory homunculus in dystonia of the hand. Ann Neurol 1998;44:828-31.

21. Byl NN, Merzenich MM, Jenkins WM. A primate genesis model of focal dystonia and repetitive strain injury: I. Learning-induced dedifferentiation of the representation of the hand in the primary somatosensory cortex in adult monkeys. Neurology 1996:47:508-20.

22. Murase N, Kaji R, Shimazu H et al. Abnormal premovement gating of somatosensory input in writer's cramp. Brain 2000;123:1813-29.

23. Frasson E, Priori A, Bertolasi L et al. Somatosensory disinhibition in dystonia. Mov Disord 2001;16:674-82. 
24. Tamura Y, Matsuhashi M, Lin P et al. Impaired intracortical inhibition in the primary somatosensory cortex in focal hand dystonia. Mov Disord 2008;23:558-65.

25. Mountcastle VB. Neural mechanisms in somesthesia. In: Mountcastle VB, editor. Medical physiology. St. Louis: Mosby;1980:348-90.

26. Mauk MD, Buonomano DV. The neural basis of temporal processing. Annu Rev Neurosci 2004;27:307-40.

27. Lidsky TI, Manetto C, Schneider JS. A consideration of sensory factors involved in motor functions of the basal ganglia. Brain Res Rev 1985;356:133-46.

28. Artieda J, Pastor MA, Lacruz F et al. Temporal discrimination is abnormal in Parkinson's disease. Brain 1992;115:199-210.

29. Pastor MA, Day BL, Macaluso E et al. The functional neuroanatomy of temporal discrimination. J Neurosci 2004;24:2585-91.

30. Delmaire C, Vidailhet M, Elbaz A, et al. Structural abnormalities in the cerebellum and sensorimotor circuit in writer's cramp. Neurology 2007;69:376-80.

31. Teo JT, van de Warrenburg BP, Schneider SA, et al. Neurophysiological evidence for cerebellar dysfunction in primary focal dystonia. J Neurol Neurosurg Psychiatry 2009;80:80-3.

32. Abbruzzese G, Berardelli A, Girlanda P, et al. Long-term assessment of the risk of spread in primary late-onset focal distonia. J Neurol Neurosurg Psychiatry 2008;79:392-6. 


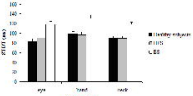

Zus abius 


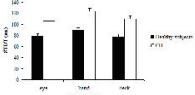

Zus abius 


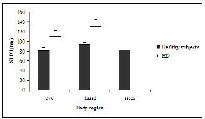




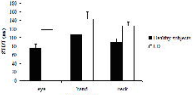

Zus abius 


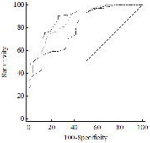

